\title{
Quality of service Management in IP Network
}

\author{
O.Chahbouni, A.Belangour, A. Namir, \\ Hassan2-Mohammedia University, Casablanca, Morocco
}

\begin{abstract}
IP network. The first objective for this solution is the satisfaction of needs appeared in several domains (Quality of service, safety, network management) by the automatization of processes for management network equipments, the simplification of data to have less interfaces numbers and more homogeneous, the decentralization of existing elements for network management, This architecture proposes the modification of Policy-based management based on definition of rolls and service contract proposed by IETF( Internet Engineering Task Force) for IP network, And thereafter we propose the generalization to assure the interoperability of various implementations (domains, equipments, participants...).

The control leans on the definition of an adequate mechanism (Application) which is going to give the possibility for every customer to define his own quality of service, his mobility and his safety, This mechanism requires the addition of a new properties to the protocol COPS(Common Open Policy Services) used before to the communication between entities of Policy-based management.
\end{abstract}

Keywords: IP network, Policy-based management, PDP, PEP, COPS

\section{INTRODUCTION}

The simplicity of the protocol IP was one of the reasons which contributed to his adoption in the majority of the computing networks, conceived at the beginning for the transfer of streams, the protocol proved very fast his limits with the appearance of new multimedia flows (Voice, phoning over IP, television Live).

The classic management for this network based largely on the model of relation agent/administrator became powerless to follow this new flow generation. The need to explore equipments as well as information models makes this classic management more and more complex front of integration for a new equipment or an option of management. This type of greedy application in resources was behind the appearance of a good many models to guarantee the QoS (Quality of Service) [1]for customers requests, Among them we find Diffserv (Differentiated Services) [2] which introduces the selective elimination based on a level of priority, or MPLS (Multiprotocol Label Switching) [3] who takes place between the data links and network coat, with replacing the ordinary commutation by etiquette commutation and adding one 'Label' which defines the road to be followed for every package, We also find the protocol Intserv (Integrated Service) [1] for principal objective the real time exchange of data customers by reservation of resources on all routers which flow will pass until the destination. But with the increase of the traffic due to the demands customers and the evolution of the network equipments, several other concerns have appeared, difficulties which result from the reassurance of the connections between terminals and edge routers, more then, this router is confronted to manage an important traffic to flow, and to memorize all these data. To answer all these questions, the implementation of politics for quality of service based on the selection of routes and the limitation of network resources for some range of customer became a necessity. So the appearance of the concepts such as "rules", " policy of centralization " in intelligent entities made its success for control and management of the network resources, we shall try in this article to bring a descriptive study to improve the architecture proposed by IETF for the management of the IP network. This solution is going to allow at the first to manage and to control the access of terminals customers within the network operator. Then, to redistribute the principal elements of PBM (Policy-based management) [4] [5] and to refine their roles. Finally, the decrease of the traffic and the exchange of data bound to the authentication of terminals and the negotiation of the services requested by the customers. This new distribution is going to bring more flexibility and mobility to equipments customers.

This control leans on the definition of an adequate management application for managing IP based networks and using the protocol COPS with different extensions, the section 2 define the notion of The PolicyBased Management (PBM) and his bases, the section 3 presents the development of roles and definition of an adequate protocol of communication, the section 4 describes the global architecture proposed and a definition of device to set up quoted in customer, the reassurance of the customer application will be developed in the section 5 . 


\section{POLICY-BASED MANAGEMENT}

The Policy-Based Management was born, further to the need of the operators for telecommunication and access providers to automate the configuration of node and diverse network equipments, this management proposes, the control of flow circulate, and the management of the network equipments.

A policy can be defined, as a set of rules establishes previously, between the customer and operator further to a discussion, or by acceptance of rules already prepared by the network operator, this policy will be afterward translated at a lower level in a network language to determine the adequate protocol and its configuration, the searches of both groups IETF (Internet Engineering Task Force) and DMTF (Distributed Management Task Force) succeeded to define a common model of information PCIM (Policy Common Information Model) [6] [7] which is going to represent the network equipments, the roles and the orders of execution, in our study which worries about the quality of service, this model allows to pass from the general study of the policy to the configuration of the router. The existent works define two standard of politics for quality of service (QoS), the first one is the QPIM (QoS Policy Information Model) [1][5] which supplied a formal representation of abstracted rules by integrating the techniques of IntServ and DiffServ, without affecting in methods of stock or protocol of access, the second extension is the QDDIM (QoS Device Datapath Information Model) [1][5] which allows with its general syntax to give a configuration of routers and the actions to execute. The general architecture of this management, consists to centralize the decisions within an intelligent entity PDP (Policy Decision Point) [1][8], he assures several features, among them one finds, the translation of the rules of politics in an understandable size for knots, the communication with the other servers to make a decision, the identification of the politics to be applied to the various PEP (Policy Enforcement Point) [1][8], this last one can be a router, a switch or even a firewall, and quite other type of intermediary between the customer and the network, its role consists in applying the decisions taken by the PDP, he can be configurable by SNMP, and by command CLI (Line Interface command).

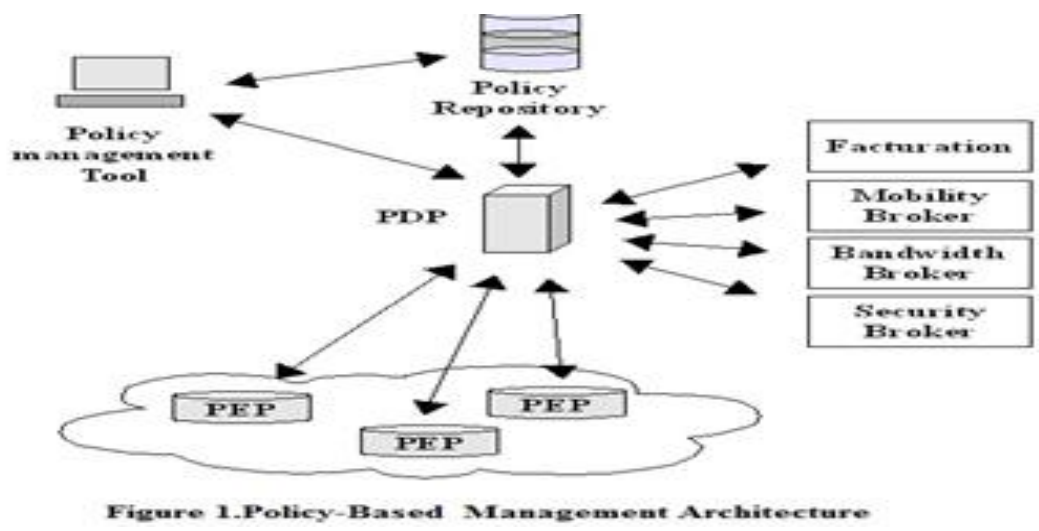

IETF proposed the protocol COPS (Common Open Policy Service) as the basic communications tool, its purpose consists in transporting the demands and in turning the politics to be applied, as well as the detection of the conflicts during the installation of a politics. COPS also interacts with two main models of the management by politics, the Outsourcing [1][5] and the Provisioning [1][5], the first one is going to delegate the decision to accept or to reject and although modify a demand of reservation of resource for a new customer to the server of politics PDP, with help of the protocol COPS-RSVP [11] extension of COPS, within a network to which the customer is not subscribed, in the favorable case, the PDP sends the configuration to be set up in the PEP bound, in his turn, this last one forwards the configuration to the customer applicant.

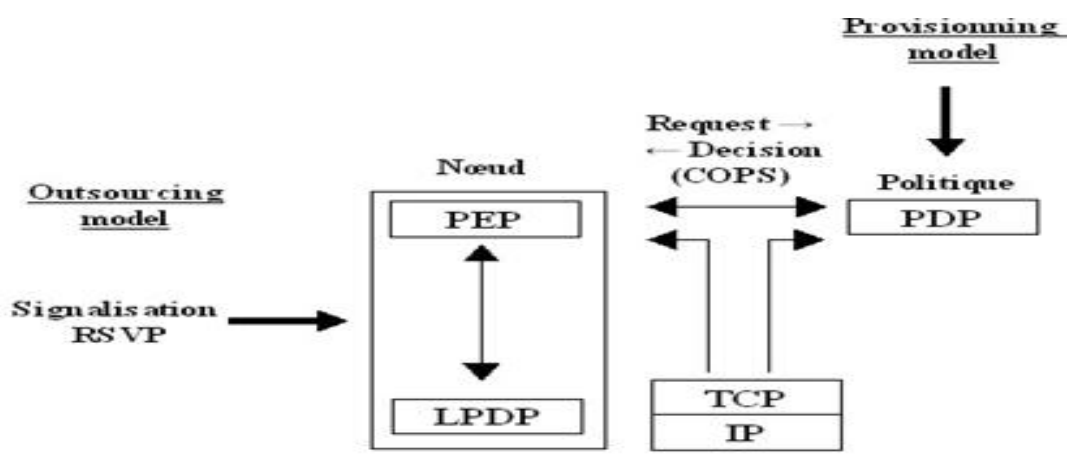

Figure 2. COPS and the Policy Based Management 
The second model consists in defining of prerequisites SLA (Level Agreements service) [13] further to a discussion between the customer and the operator, this service contract defines the responsibilities of the supplier in term of rate of availability, degree of stability of networks, and method of calculation, it will be afterward translated into a technical specification SLS (Level Specification service) [14] to memorize in the databases of the operator, which will be requested in the future during the sending of the customer flow in the entrance of the network, this model is often associated with the technique DiffSev in which streams are classified according to several classes, to set up these various configurations of management of the QoS, Provisioning uses the extension COPS-PR [12] for the transfer of the data of politics which take the name of PIB (Policy Information Base) [1][8].

\section{DEFINITION OF THE PROTOCOL AND THE DEVICE FOR MANAGEMENT}

After the microscopic study of the model centralized the Policy-Based Management and the distribution of entities, we tried to improve this model so as to decrease the traffic bound to the centralization of the decisions within the PDP, and to give more flexibility to the customer to manage his own account, $\mathrm{We}$ define at the beginning a new notion LAS (level of available service) and we describe the scenarios possible during the connection of a new customer or already existing in the data base of the operator.

At the first and before any communication with a new customer, the PDP makes a calculation on these databases in functions of existing contract SLA ( service-level agreement ) defined between operator and customer, with this study we will go establish levels of available service (LAS) which determines the available configuration which the network operator can supply and offer during a next demand. After this determination of the LAS, they will be afterward, forwarded quite benefit from asynchronous mode of the PDP in all the PEP

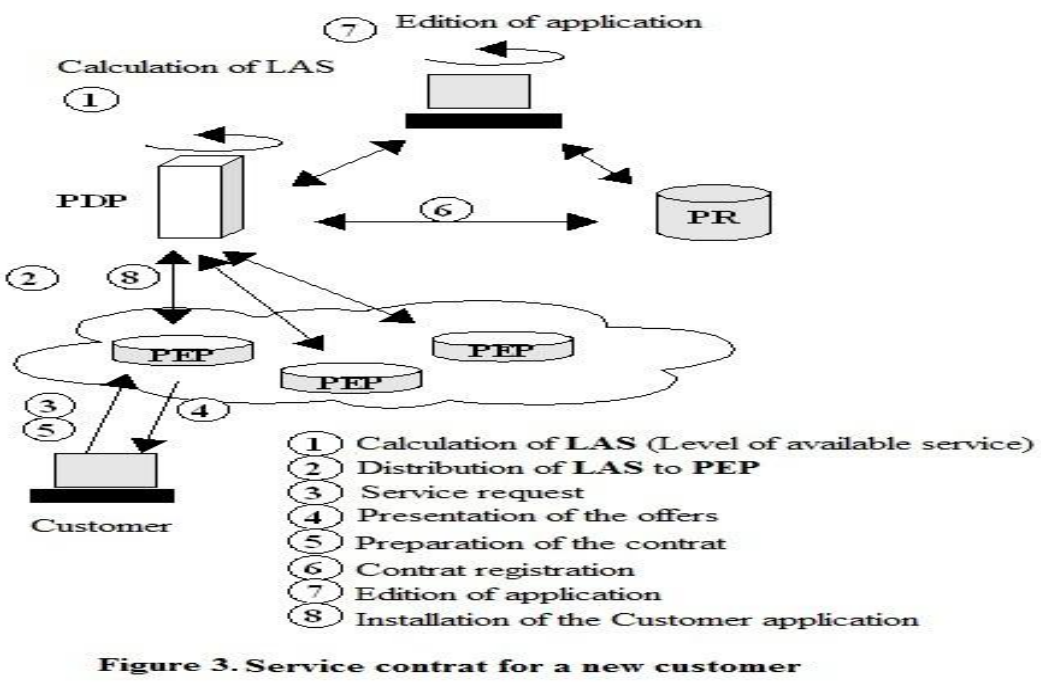

which are bound to him. In the connection of a customer in the network operator, we can distingue between two modes of connection, the first one is for a new customer who requires quality of service for these demands, a phase of negotiation will be established between the applicant and the PEP only without referring to the PDP, the PEP offers a proposal to the customer by basing itself on the LAS sent previously by the PDP, after the negotiation of both participants, the PEP sends to the PDP the project of the established contract which is the report of negotiation, and asks the recording this new customer in database PR (Policy Repository) [1][8], also the creation of a subscribed account. The PDP converts the rules of politics in an adapted form named PIP(Policy Information Point) which is the source off attribute values, this translation as well as the information of the account and the contract elaborated for this customer will be afterward translated in an adequate mechanism generated by the PDP and sent at the new subscriber.. This shape of management became possible thanks to the improvement of the PEP by the integration of the component LPDP (Local Policy Decision Point) [1][8] who plays a role of local mask of the politics without interacting with the PDP, This last guard always the property to control and oversee the network and all the knots thanks to its communication with these servers local or distant as the server policy repository in whom the PDP is going to make the search on the rules of politics or the server of bandwidth who manages the available bandwidth in the network, We notice that the load of negotiation will be decentralized towards the PEP and that a new mechanism is defines in an adaptable format for every customer that we shall explain later.The addition of every new customer activates the update of the base of datum, as well as the calculation of the LAS and the distribution of this information to all the PEP / 
LPDP. The PEP can appeal at any time to the PDP to react in front of a situation for which it does not arrange adequate rules, it is so responsible for the cancellation of any demand become unacceptable continuation has a customer modification.

This shape of communication requires the use of the basic protocol COPS, this last one uses a model customer / server, to whom the PEP sends messages of decision, the PDP is going to answer as a consequence by messages containing the taken decisions.

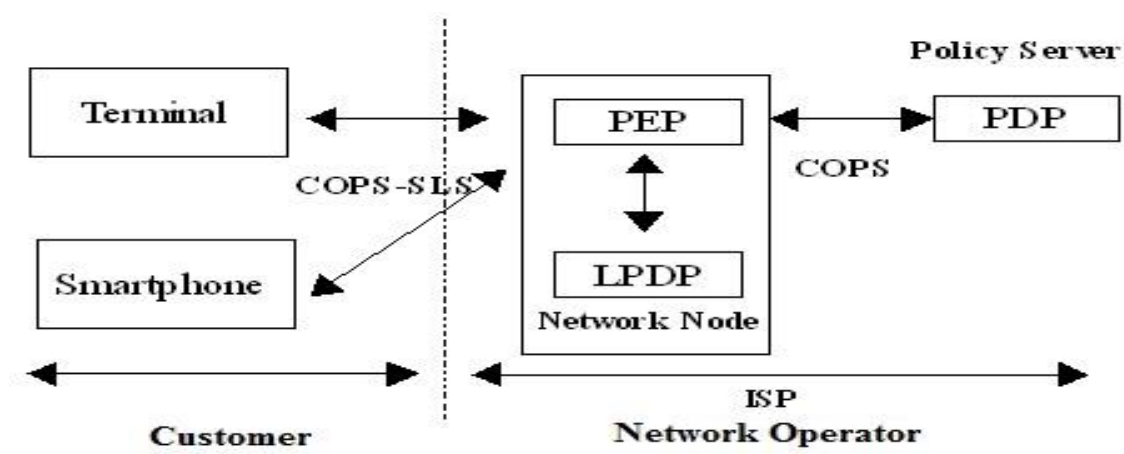

Figure 4. Distribution of protocol COPS

In our approach the use of COPS will be spread for the negotiation between the customer and the PEP, without needing to refer to the server of politics PDP, the extension COPS-SLS [9][10] proposed in June 2001 by LIP6 and ENST will be requested, We defined at the beginning the basic protocol and we shall explain afterward its improvement in our proposal, this protocol in for main purpose the negotiation of the politics between the PEP and the PDP, he can appear as a communications tool inter and intra domain, In the model COPS-SLS proposed previously, the negotiation is dynamic between the customer station and the server of the politics, this automation gives to the customer the ease to seize its SLS directly with its ISP (Internet Provider service). This protocol includes two phases, the phase of configuration which determines the way of negotiating, and the phase of negotiation, which takes care of the exchange of the information necessary for the definition of the SLS between the PEP and the PDP for the establishment of the contract, To succeed has this shape of management, we pass by the installation of the way of negotiating the SLS with PEP while benefiting from the provisioning model by the PDP, after this negotiation, the wished SLS will be sent to the PDP, This last one makes its decision to accept or to reject the demand or to propose another contract, the report of customer installation is sent to the PDP, after check of the report, the PDP signs the contract and the customer benefits from the level of service, in the opposite case no contract is established, in our solution the protocol COPS-SLS will be adapted the way that negotiation is made between the customer and the PEP, the authorities of classes PIP, Named ClientSI used for the phase of configuration, and Signaled ClientSI used for the phase of negotiation are encapsulated in the object ClientsSI of the protocol COPS-SLS.

We were able to adapt this protocol for a communication express between the customer and the PEP thanks to its flexibility for the dynamic negotiation of the SLS. after the agreement of the PDP a device which allows to identify the customer as well as its contract will be edited by the operator and to install at the customer terminal. In the case of a connection of a customer already registered in the database PR using any support(fixed terminal or mobile, Smart phone), the device installed previously at the customer since its first connection is going to allow him to send the flow without negotiation or interrogation of the router to border, This last one as well as everything the knots of the network will be ready has to receive these flows of data negotiated previously in the SLA-SLS, the device installed at the customer allow him to be mobile and more flexible for the exchange of streams, with this mechanism the customer will not need to connect in a specific router to emit these data.

\section{GLOBAL ARCHITECTURE}

The main objective was proposed in this article, it is the redistribution of the roles of the entities that constituting the management by politics, and the decentralization of spots towards the periphery of the network for reasons already evoked several times, the second objective the decrease of the traffic bound has the customer identification and the number of protocols used in the network domain by the use of the protocol COPS-SLS adapted to our architecture. 


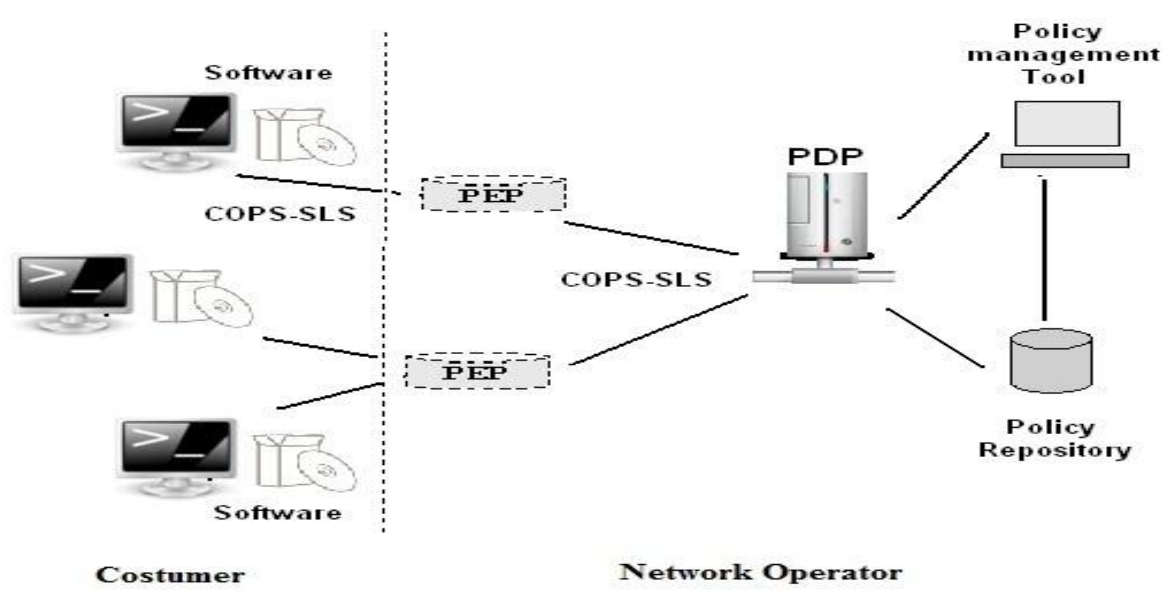

Figure 5. Global Architecture

To complete this architecture, the plan which we quoted previously in the previous section is translated in application for every type of customer equipment, the setting-up of a software which is going to contain the customer identity, by the identification and the authentication of equipments customer, as well as the negotiated contract, this application will be strengthened by algorithms of control, the customer cannot modify them. For the identification of the user, the customer application contains the algorithm of identification which allows to verify the authenticity of this customer in a server radius at the operator, through the protocol COPSSLS.

\section{CONCLUSION}

This article introduces new concepts and new notions which allow the change of the classic management classic, the decentralization of spots and redistribution of the roles as well as the mobility of the customer terminal are the main profits of our approach, we were able to adapt the protocol COPS-SLS dedicated to the base for the dynamic negotiation of the contract, has our solution of a way negotiated the SLA between the customer and the PEP directly and also the authentication of costumer wherever he go with the help of the application.

\section{REFERENCES}

[1]. Jean-Louis Melin, Qualité de service sur IP, Ethernet, Frame Relay et ATM, Eyrolles 7 mars 2001

[2]. An Architecture for Differentiated Service RFC 2475, Assured Forwarding PHB Group RFC 2597, Configuration Guidelines for DiffServ Service ClassesRFC 4594,RFC 2597, Internet Engineering Task Force (IETF).

[3]. Mehmet BEYAZ, Multi-Protocol Label Switching, Next Generation Networks, TTG International, L.T.D. 2008.

[4]. IETF, RFC 3198 Terminology for Policy-Based Management, http://www.ietf.org/rfc/rfc3198, Nov 2001.

[5]. GUY PUJOLLE «Les Réseaux», Best of Eyrolles, 5ème EDITION, groupe Eyrolles 2006.

[6]. Policy Core Information Model, http://rfc-ref.org/RFC-TEXTS/3060/kw-pcim.html. Common Information Model (CIM) Schema, version 2.x. DMTF,www.dmtf.org/ standards/standard cim.php.

[7]. Jean-Christophe Martin, Policy Based Networks, Sun BluePrints Online - Octobre 1999.

[8]. T.M.T. Nguyen, G. Pujolle, N. Boukhatem, and D. Gaïti, "Contrôle des réseaux IP fixes et Mobiles", ;in Proc. RIVF, 2003, pp.127-132.

[9]. T. Nguyen, N. Boukhatem and G. Puiolle, G., COPS-SLS usage for dynamic policy based QoS management over heterogeneous IP networks, IEEE Networks, Volume17, (3), May-June, 2003.

[10]. Policy Quality of Service (QoS) Information Model. http://tools.ietf.org/html/rfc3644

[11]. COPS Usage for Policy Provisioning (COPS-PR). https://tools.ietf.org/html/rfc3084

[12]. Dinesh C. Verma "Service Level Agreements on IP Networks",IBM T. J Watson Research Center, USA.

[13]. D. Grossman, New Terminology and Clarifications for DiffServ, RFC 3260,April 2002. 